\title{
Synovial Sarcoma Oropharynx - A Case Report and Review of Literature
}

\author{
Nagendra P. B. Kadapa $\cdot$ L. Sudarshan Reddy $\cdot$ Ranganatha Swamy $•$ Kumuda • \\ M. Vishnu Vardhan Reddy • L. M. Chandra Sekhara Rao
}

Received: 13 August 2013 / Accepted: 30 December 2013 /Published online: 15 January 2014

(C) Indian Association of Surgical Oncology 2014

\section{Introduction}

Synovial sarcoma is a rare malignant tumor that derives from a mesenchymal precursor stem cell that is unrelated to mature synovial tissue. It is primarily a disease of young adults typically arising in the deep soft tissues of the extremities, most commonly, the lower thigh-knee region. Reports in the literature have underscored the occurrence of this tumor in unusual sites, many seemingly distant from synovium-lined spaces, particularly, the abdominal wall [1] and head and neck region. The vast majority of this latter group, mostly case reports, were confined to the cervical and parapharyngeal region [2-8]. In this article, we report a case of synovial sarcoma that originated in oropharynx which is extremely rare. This is the first of its kind at our institute.

\section{Case Report}

A 40-year-old man presented with a history of foreign body sensation in the throat mainly on the left side \& progressively worsening dysphagia and nasal regurgitation. He did not have other symptoms relating to the ear, nose and throat. He had no

N. P. B. Kadapa $\cdot$ L. S. Reddy $\cdot$ R. Swamy $\cdot$ Kumuda

M. V. V. Reddy $\cdot$ L. M. C. S. Rao

Department of Surgical Oncology, Basavatarakam Indo-American

Cancer Hospital, Banjarahills, Hyderabad, Andhra Pradesh, India

\section{S. Reddy $(\square)$}

Osmania Medical College/Govt. ENT Hospital, Koti,

Hyderabad 500095, Andhra Pradesh, India

e-mail: drlsudarshanreddy9@gmail.com

L. S. Reddy

e-mail: aoiapstatebranch@gmail.com history of a loss of weight or appetite. He had given up smoking 7 years earlier.

Examination revealed the presence of a pedunculated mucosal lesion projecting into oropharynx arising from just behind the posterior pillar of tonsil on left side. No cervical lymphadenopathy was noted. Findings on the remainder of the ENT examination were normal. A lateral neck x-ray demonstrated an abnormal protrusion of soft tissue from the lateral wall of oropharynx on left side.

The patient underwent a pharyngoscopy showing well demarcated globular mass of $4 \times 3 \mathrm{~cm}$ arising from left lateral pharyngeal wall just behind posterior pillar of tonsil. Computed Tomography scan revealed a lobulated homogenously enhancing soft tissue density mass lesion in left lateral pharyngeal wall extending from tip of epiglottis to hyoid bone. The mass was removed as an excisional biopsy by transoral endoscopic assisted approach under general anaesthesia (Figs. 1 and 2).

Histo-pathologic examination showed biphasic tumour with slit like spaces lined by epithelial cells and Spindle cells. Epethelial cells were cuboidal and pale with vesicular nuclei and spindle cells were in fascicles and bundles, closely approximated to epithelial and glandular spaces (Fig. 3) consistent with synovial sarcoma/carcinosarcoma. Excision of lesion was deemed complete. Immunohistochemistry highlighted spindle cells strongly expresses for vimentin, focal weak cytoplasmic positive for $b c l-2$, pancytokeratin positive in epithelial cells and CD99 was negative consistent with Synovial sarcomama/ Carcino sarcoma. It was subjected to polymerase chain reaction technique for $\mathrm{t}(\mathrm{X} ; 18)$ (p11.2; q11.2) SYT-SSX analysis. Results turned positive for $S S X 1$ and SYT genes and confirmed as biphasic synovial sarcoma [9].

Patient received adjuvant radiotherapy 66 Gy in 33 fractions. Case was followed up for 3 years at regular intervals for both local and metastatic work up and is free of any symptoms. Residual or recurrent mass ruled out by VLS. 

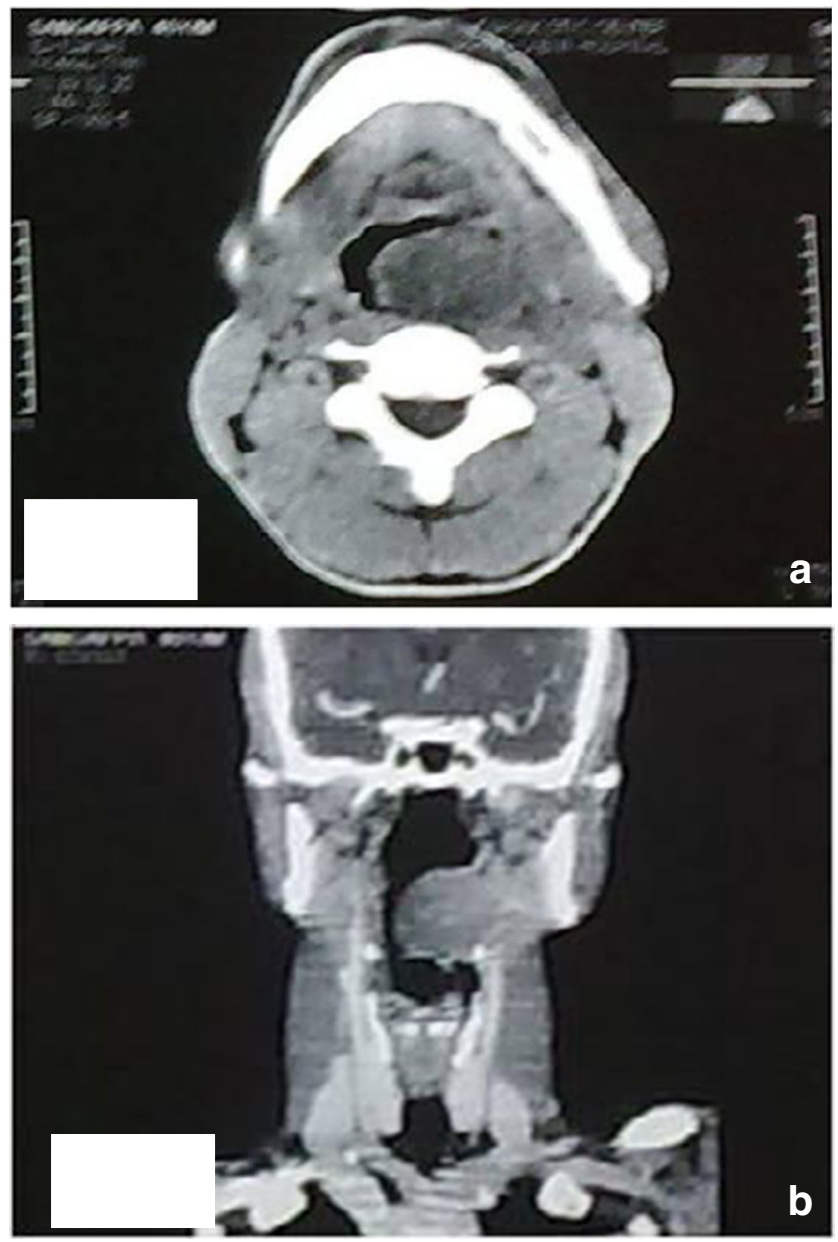

Figs. 1 and 2 CT scan showing lobulated homogenously enhancing soft tissue density mass lesion in left lateral pharyngeal wall extending from level of epiglottis to hyoid bone in axial view (I) and coronal view (2)

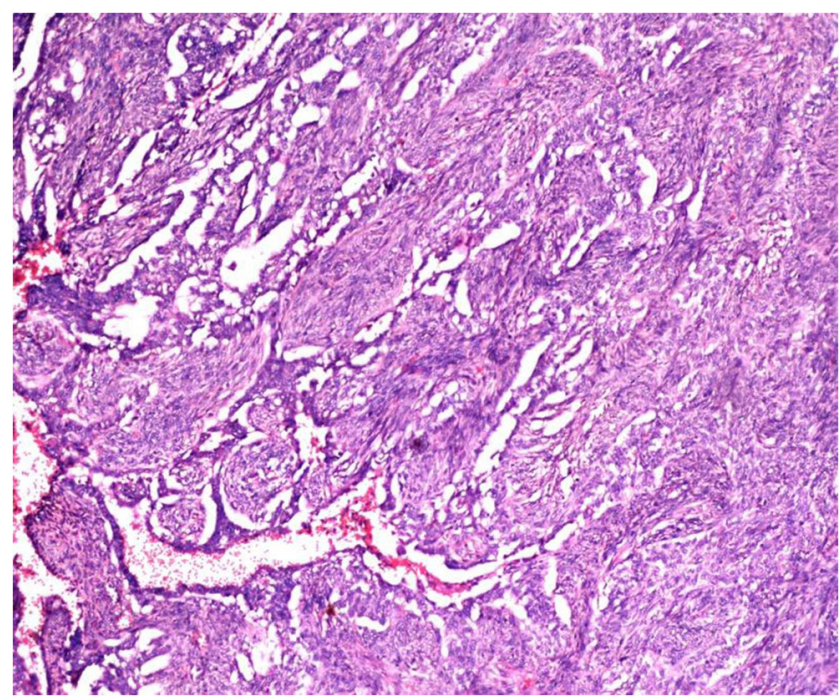

Fig. 3 HPE: biphasic tumour with slit like spaces lined by epithelial cells and spindle cells in fascicles and bundles, closely approximated to epithelial and glandular spaces

\section{Discussion}

Synovial sarcoma is a malignant soft-tissue neoplasm that arises from primitive pluripotential mesenchymal cells near to or remote from articular surfaces. Other terms used to describe this entity are synovial cell sarcoma and malignant synovioma $[9,10]$. Although the term synovioma implies a benign condition, further investigations have exposed the aggressive nature of this neoplasm and eventually led to its classification as a high-grade tumor by the American Joint Committee on Cancer [11].

Synovial sarcomas account for 8 to $10 \%$ of all sarcomas [2]. Although these tumors occur predominantly in the extremities, they are also known to arise in the head and neck region [9]. Since the first description of a head and neck synovial sarcoma of hypopharynx by Jernstrom [3] in 1954, fewer than 90 cases have been reported in the literature, and they account for 3 to $10 \%$ of all reported cases [12].

The most common site of synovial sarcoma of the head and neck is the hypopharynx $[4,10]$. These tumors have been encountered in other areas of the head and neck as well, including prevertebral and parapharyngeal areas, pharyngeal, laryngeal, nasopharynx, soft palate, tongue, maxillofacial region, mandible corner, sternoclavicular region, scapular region and the cervical oesophagus [5]. Synovial sarcoma classically affects patients between the ages of 15 and 40 years [6], and the proportion of male-to-female patients is $3: 2$ [7]. Patients with synovial sarcoma of the head and neck tend to be younger than those who have such tumors in the extremities $[5,12]$.

The tumor usually appears as an asymptomatic mass until it attains sufficient volume to cause pressure effects on neighbouring structures. This process is usually more rapid in patients with head and neck synovial sarcoma than in those whose tumor is in an extremity. Lockey reported that patients with head and neck tumors were diagnosed an average of 20 months earlier than those with extremity tumors [12]. Head and neck synovial sarcomas have better prognosis because of their slow growth rate and lower rate of lymph node metastasis. Pai et al. reported that survival in cases of neck disease was higher than that in cases of limb tumors [8]. On the other hand, Kartha and Bumpous found that the 5-year survival rate for patients with head and neck synovial sarcoma was $40 \%$, which is substantially lower than the $60 \% 5$-year survival rate reported in the literature for synovial sarcoma at all other sites [13].

Histologically, three variants of synovial sarcoma have been described: biphasic, monophasic fibrous, and monophasic epithelial. Although monophasic forms of synovial sarcoma have been reported, its primary histopathologic feature is biphasic cellularity (Fig. 3) [14]. Microscopically, the tumor is made up of epithelial and sarcomatous components. Epithelial areas are present in the form of glands lined by cuboidal or columnar cells 
or as solid nests as was seen in this case. Sarcomatous component is made up of hypercellular spindle cells with fibroblast-like cells or hemangiopericytoma-like areas. Extensive sampling is required to display epithelial features. Synovial sarcoma with only spindle cells or purely glandular component is called monophasic synovial sarcoma. Calcifying Synovial sarcoma is another subtype, which has extensive calcification and carries a much better prognosis. Histologically, no tumour in the body resembles biphasic Synovial sarcoma. However, monophasic Synovial sarcoma simulates fibro sarcoma, malignant peripheral nerve sheath tumour and hemangiopericytoma. Reticulin stain highlights the imperceptible clusters of epithelial cells. Immunohistochemically $\mathrm{S}-100, \mathrm{CD}-99$, bcl-2 and vimentin positivity is seen in the spindle cells of Synovial sarcoma. Cytokeratin expression is seen in $90 \%$ of all Synovial sarcoma both in the epithelial and a few cells of the sarcomatous component. Specific expression of Cytokeratin subtypes 7 and 19 along with EMA is observed, and expression of epithelial markers in the epithelial as well as sarcomatous element excludes the possibility of Ewing's sarcoma and Malignant peripheral nerve sheath tumour, which may also show positivity for CD-99 and S-100 on immunohistochemistry in the spindle cell areas. Ultrastructurally, true glandular epithelial features are seen in epithelial areas. Spindle cell areas also show subtle epithelial features. The $t(x ; 18)(p 11 ; q 11)$ is the cytogenetic hallmark of synovial sarcoma. FISH and RT-PCR have been employed for the rapid diagnosis of Synovial sarcoma [14].

Preoperatively, computed tomography and magnetic resonance imaging are the investigations of choice to determine the location and extent of the tumor.

Small number of patients with synovial sarcoma of the head and neck precludes us from drawing firm conclusions with respect to a precise treatment protocol. The primary mode of treatment is wide surgical excision [10] that includes a normalappearing tissue cuff in order to obtain tumor-negative margins. However, this cannot always be accomplished in head and neck area because of the proximity of the tumor to vital structures. The results of multimodality treatment appear to be better than those seen with any single-modality treatment or with any form of salvage therapy $[12,15]$. The role of radiotherapy is confined to reducing the risk of local recurrence following resection; radiation does not improve long-term survival [16]. The role of chemotherapy is controversial [17], although Lenoir et al. [18] reported a dramatic regression in tumor size in a 15-month-old patient with a retropharyngeal synovial sarcoma after treatment with a combination of ifosfamide, vincristine, and actinomycin D. The future role of both radiotherapy and chemotherapy in the management of synovial sarcoma needs to be determined by a prospective study.

Favourable prognostic indicators include an early diagnosis, small tumor size $(<5 \mathrm{~cm})$, younger age $(<20$ years), distal location of tumor, mitosis $<15 / 10 \mathrm{HPF}$, calcification and a wide surgical excision $[16,17,19]$. However, necrosis, poorly differentiating areas and aneuploidy are worse prognostic indicators [14].

We presented this case because of its rarity in its presentation as an oropharyngeal synovial sarcoma, not more than ten cases reported in the world literature as far as our knowledge [19-23].

\section{References}

1. Hale JE, Calder IM (1970) Synovial sarcoma of the abdominal wall. Br J Cancer 24:471-474

2. Carrillo R, Rodriguez-Peralto JL, Batsakis JG (1992) Synovial sarcomas of the head and neck. Ann Otol Rhinol Laryngol 101:367-370

3. Jernstrom P (1954) Synovial sarcoma of the pharynx: report of a case. Am J Clin Pathol 24:957-961

4. Dei Tos AP, Dal CP, Sciot R et al (1998) Synovial sarcoma of the larynx and hypopharynx. Ann Otol Rhinol Laryngol 107:1080-1085

5. Doval DC, Kannan V, Mukherjee G et al (1997) Synovial sarcoma of the neck. Eur Arch Otorhinolaryngol 254:246-250

6. Onerci M, Sarioglu T, Gedikoglu G et al (1993) Synovial sarcoma in the neck. Int J Paediatr Otorhinolaryngol 27:79-84

7. Gatti WM, Strom CG, Orfei E (1975) Synovial sarcoma of the laryngopharynx. Arch Otolaryngol 101:633-636

8. Pai S, Chinoy RF, Pradhan SA et al (1993) Head and neck synovial sarcomas. J Surg Oncol 54:82-86

9. Cihak RA, Lydiatt WM, Lydiatt DD, Bridge JA (1997) Synovial sarcoma of the head and neck: chromosomal (translocation)(X;18)as a diagnostic aid. Head Neck 19:549-553

10. Mamelle G, Richard J, Luboinski B et al (1986) Synovial sarcoma of the head and neck: an account of four cases and review of the literature. Eur J Surg Oncol 12:347-349

11. Russell WO, Cohen J, Enzinger F et al (1977) A clinical and pathological staging system for soft tissue sarcomas. Cancer 40:1562-1570

12. Lockey MW (1976) Rare tumors of the ear, nose and throat: synovial sarcoma of the head and neck. South Med J 69:316-320

13. Kartha SS, Bumpous JM (2002) Synovial cell sarcoma: diagnosis, treatment, and outcomes. Laryngoscope 112:1979-1982

14. Muhammad Ashraf S, Sajid M, Nadira M, Muhammad Tahir K, Zunera A (2008) J Coll Phys Surg Pak 18(11):713-715

15. Patel SC, Silbergleit R, Talati SJ (1999) Sarcomas of the head and neck. Magn Reson Imaging 10:362-375

16. Amble FR, Olsen KD, Nascimento AG, Foote RL (1992) Head and neck synovial cell sarcoma. Otolaryngol Head Neck Surg 107:631-637

17. Yokoyama K, Shinohara N, Kondo M, Mashima T (1995) Prognostic factors in synovial sarcoma: a clinicopathologic study of 18 cases. Jpn J Clin Oncol 25:131-134

18. Lenoir P, Ramet J, Goossens A et al (1991) Retropharyngeal synovial sarcoma in an infant: report of a case and of its response to chemotherapy; review of the literature. Pediatr Hernatol Oncol 8:45-52

19. Kokot N, Mazhar K, O'Dell K, Huang N, Lin A, Sinha UK (2013 Jun) Transoral robotic resection of oropharyngeal synovial sarcoma in a pediatric patient. Int J Pediatr Otorhinolaryngol 77(6):1042-1044

20. Herrero Laso JL, Varela DJ (1998) Oropharyngeal synovial sarcoma. Report of one case. An Otorrinolaringol Ibero Am 25(4):353-359

21. Genest P, Kim TH, Katsarkas A, Jothy S (1983) Calcified synovial sarcoma of the oropharynx. Br J Radiol 56(668):580-582

22. Vogel U, Wehrmann M, Eichhorn W, Bultmann B, Stiegler M, Wagner W (2010) Molecular and clinicopathological findings in a tonsillar synovial sarcoma. A case study and review of the literature. Head Neck Pathol 4:257-260

23. Mir-Abedy M (1962) Considerations of the base of the tongue and its tumors. Ann Otolaryngol Chirurg Cervico-Far 791:547-561 